\title{
Angular control of optical cavities in a radiation-pressure-dominated regime: the Enhanced LIGO case
}

\author{
Katherine L. Dooley, ${ }^{1,7, *}$ Lisa Barsotti, ${ }^{2}$ Rana X. Adhikari, ${ }^{3}$ Matthew Evans, ${ }^{2}$ Tobin T. Fricke,,${ }^{4,7}$ \\ Peter Fritschel, ${ }^{2}$ Valera Frolov, ${ }^{5}$ Keita Kawabe, ${ }^{6}$ and Nicolás Smith-Lefebvre ${ }^{2,3}$ \\ ${ }^{1}$ University of Florida, P.O. Box 118440, Gainesville, Florida 32611, USA \\ ${ }^{2}$ LIGO Laboratory, Massachusetts Institute of Technology, Cambridge, Massachusetts 02139, USA \\ ${ }^{3}$ LIGO Laboratory, Division of Physics, Math, and Astronomy, California Institute of Technology, \\ Pasadena, California 91125, USA \\ ${ }^{4}$ Louisiana State University, Baton Rouge, Louisiana 70803, USA \\ ${ }^{5}$ LIGO_Livingston Observatory, Livingston, Louisiana 70754, USA \\ ${ }^{6}$ LIGO—Hanford Observatory, Richland, Washington 99354, USA \\ ${ }^{7}$ Albert-Einstein-Institut, Max-Planck-Institut für Gravitationsphysik, D-30167 Hannover, Germany \\ *Corresponding author: katherine.dooley@ligo.org
}

Received September 19, 2013; accepted October 8, 2013;

posted October 31, 2013 (Doc. ID 197891); published November 27, 2013

\begin{abstract}
We describe the angular sensing and control (ASC) of $4 \mathrm{~km}$ detectors of the Laser Interferometer Gravitational-Wave Observatory (LIGO). Enhanced LIGO, the culmination of the first generation LIGO detectors, operated between 2009 and 2010 with about $40 \mathrm{~kW}$ of laser power in the arm cavities. In this regime, radiation-pressure effects are significant and induce instabilities in the angular opto-mechanical transfer functions. Here we present and motivate the ASC design in this extreme case and present the results of its implementation in Enhanced LIGO. Highlights of the ASC performance are successful control of opto-mechanical torsional modes, relative mirror motions of $\leq 1 \times 10^{-7} \mathrm{rad} \mathrm{rms}$, and limited impact on in-band strain sensitivity. (c) 2013 Optical Society of America OCIS codes: $\quad$ (120.2230) Fabry-Perot; (120.3180) Interferometry; (140.3518) Lasers, frequency modulated;

(350.1270) Astronomy and astrophysics. http://dx.doi.org/10.1364/JOSAA.30.002618
\end{abstract}

\section{INTRODUCTION}

Over the last decade, a worldwide network of ground-based laser interferometers [1] has been constructed and operated in pursuit of the first direct detection of gravitational waves (GWs). The U.S. Laser Interferometer Gravitational-Wave Observatory (LIGO) [2] operates detectors in Livingston, Louisiana, and Hanford, Washington, each consisting of a suspended Michelson interferometer with 4 km Fabry-Perot arm cavities. These detectors attained their best sensitivity yet during the most recent scientific data-taking run, known as S6, which took place between July 2009 and October 2010 in a configuration called "Enhanced LIGO" [3-6]. Enhanced LIGO featured several improvements with respect to the earlier Initial LIGO configuration (2001-2007). One of the critical upgrades was the increase in the laser power circulating inside the arm cavities by about a factor of four. The $40 \mathrm{~kW}$ of laser power stored in the Enhanced LIGO cavities greatly complicated the relative alignment of the interferometer mirrors. For the laser interferometer to operate properly, its mirrors must be aligned to each other with a relative rms misalignment not larger than about a tenth of a microradian. Meeting this stringent requirement is particularly challenging in the presence of radiation-pressure effects.

Radiation pressure exerts torque on suspended mirrors, adding to the fixed restoring torque of the suspension. The possibility of this torque to destabilize optical cavities was first recognized in 1991 by Solimeno et al. [7]. By 2003, it was clear in the LIGO community that the effect of radiation pressure on angular dynamics was relevant for LIGO [8], and the full details of the effects were described by Sidles and Sigg in 2006 [9]. Fan et al. measured the predicted opticalmechanical torsional stiffness at the Gingin Facility in Australia [10], Driggers demonstrated its effect at the Caltech $40 \mathrm{~m}$ prototype [11], and Hirose et al. showed that, although the optical torque in Initial LIGO (about $10 \mathrm{~kW}$ of laser power circulating in the Initial LIGO arm cavities) was measurable and similar in magnitude to the suspension-restoring torque, it was not yet significant enough to require a change to the angular controls [12]. In this paper, we show the effect of optical torque in the Enhanced LIGO interferometers and also present the design concept and implementation of an angular sensing and control (ASC) scheme, which allowed us to operate an interferometer with angular mechanics dominated by radiation pressure.

Two of the authors (Barsotti and Evans) created a numerical model of the ASC for Enhanced LIGO that specifically included radiation-pressure torque [13]. They showed that, in principle, the radiation-pressure torque can be controlled without detrimental consequences to the sensitivity of the detector. The proposed solution rotates the control basis to one that naturally represents the eigenmodes of mirror motions coupled by radiation pressure. We implemented this control 
scheme on the Enhanced LIGO interferometers with up to $40 \mathrm{~kW}$ of circulating power, successfully controlling the angular degrees of freedom in the presence of radiation-pressure instability. The demonstrated solution meets the LIGO requirements and is extensible to the next generation of LIGO detectors currently under construction, Advanced LIGO.

The interferometer layout and the control scheme are introduced in Section 2. Section 3 presents the modified design after a review of the physics of radiation-pressure-induced torque on the mirrors. This section also highlights a direct measurement of the opto-mechanical modes that are controlled. Section 4 presents the results of using the new alignment control scheme at high laser powers, including residual mirror motion and noise performance. Key differences and implications for Advanced LIGO are outlined in Section 5 . Section 6 provides a summary. All data presented are from the Livingston Observatory; results from the Hanford Observatory are similar.

\section{EXPERIMENTAL SETUP}

Each LIGO detector is a power-recycled Fabry-Perot Michelson laser interferometer featuring suspended test masses (mirrors) in a vacuum. A stabilized laser beam (with a wavelength of $1064 \mathrm{~nm}$ ) is directed to the interferometer, whose two arm lengths are set to maintain nearly destructive interference of the recombined light at the Michelson (dark) antisymmetric port. An appropriately polarized GW differentially changes the arm lengths, producing a signal at the antisymmetric port proportional to the GW strain. The test masses are suspended by a single loop of steel wire to provide isolation from ground motion, as depicted in Fig. 1. Each mirror is equipped with five magnet-coil actuators to control the mirror's longitudinal and angular position. Furthermore, the carrier laser field is phase-modulated by an electro-optic modulator at 24.4 and $61.1 \mathrm{MHz}$ to generate sidebands for use in a modulation-demodulation technique of sensing the interferometer's longitudinal and angular degrees of freedom.

There are several reasons why the interferometer's mirrors must be actively aligned: to maximize optical power coupling; to suppress motion from external disturbances; and to counteract a static instability at high laser power.

The requirements for how much residual motion is tolerable $[14,15]$ stem from the mechanisms by which misalignment couples to strain sensitivity. The most significant coupling of angular motion to cavity length occurs when the beam spot is off-center from the mirror's axis of rotation. The combination of mirror angular motion $\theta_{\text {mirror }}(f)$ and beam spot motion on the test masses $d_{\text {spot }}(f)$ changes the length of the arms by

$$
\Delta L(f)=d_{\mathrm{spot}}^{\mathrm{RMS}} \times \theta_{\text {mirror }}(f)+\theta_{\text {mirror }}^{\mathrm{RMS}} \times d_{\text {spot }}(f)
$$

and results in an increase in the sensed longitudinal motion. The relevant quantities for describing the mirror's motion are its root-mean-square (rms) and in-band (audio frequency) noise. It is worth noting that once all of the interferometer cavities are brought to resonance, and the DC pointing no longer contributes to the rms, the rms is dominated by the pendular motion.

There are additional mechanisms by which misalignment affects displacement sensitivity. First, a high-order effect arises because misalignments affect power build-up quadratically, which in turn modulates the noise floor in the shotnoise-limited regime. A second mechanism results as a side effect of having active angular alignment. Due to imperfections in the actuators, there will always be a small amount of longitudinal acutation along with the desired angular actuation.

External disturbances that cause misalignment include seismic noise, pitch/yaw mode thermal noise, length-to-angle

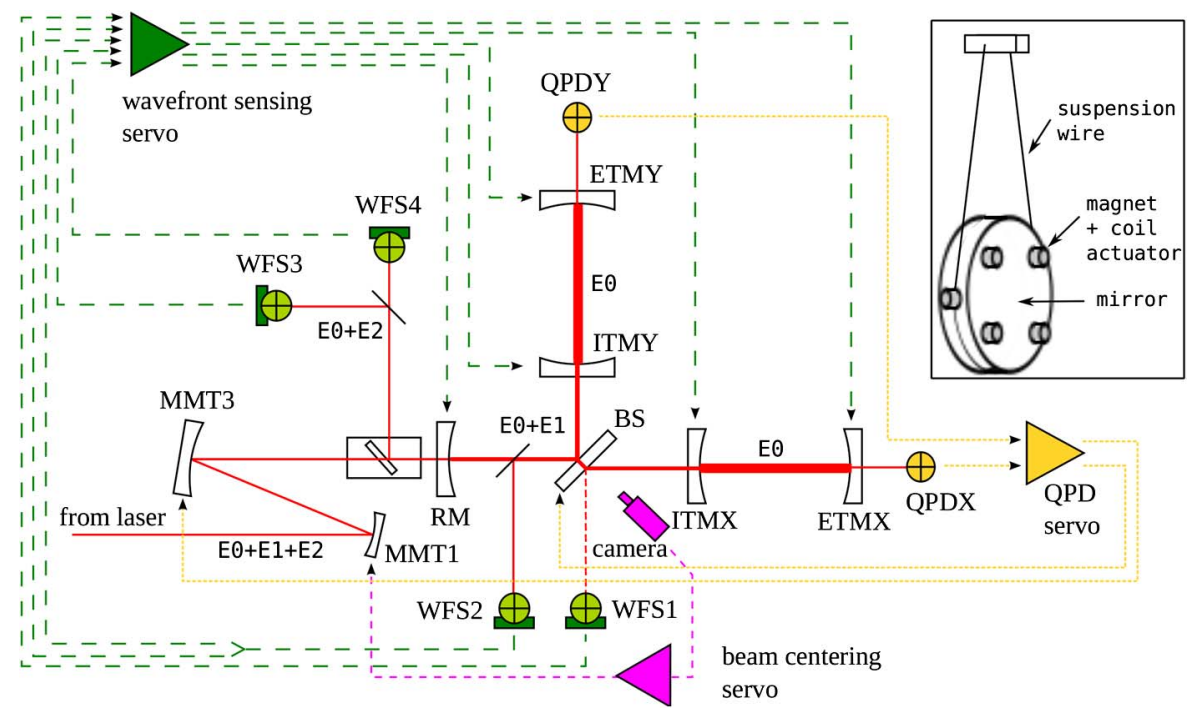

Fig. 1. Power-recycled Fabry-Perot Michelson interferometer layout, with ASC system superimposed. The eight actively aligned mirrors (ITMs, ETMs, MMTs, BS, and RM) and the ASC sensors (WFS, QPDs, and camera) are shown. All additional optics are omitted for simplicity. The QPD and beam-centering servos provide drift control on minute time scales. The wavefront sensing (WFS) servo maintains the alignment of the interferometer mirrors with respect to each other up to several Hz. Both I and Q phases are used from WFS2, whereas only one quadrature is read out from each of the other WFS. The carrier field is $E 0$, and sideband fields $E 1$ and $E 2$ are, respectively, resonant and nonresonant in the power-recycling cavity. All test masses are suspended as single-stage pendula, as depicted in the upper right corner, and are outfitted with magnet-coil actuators to control angular and longitudinal degrees of freedom. 


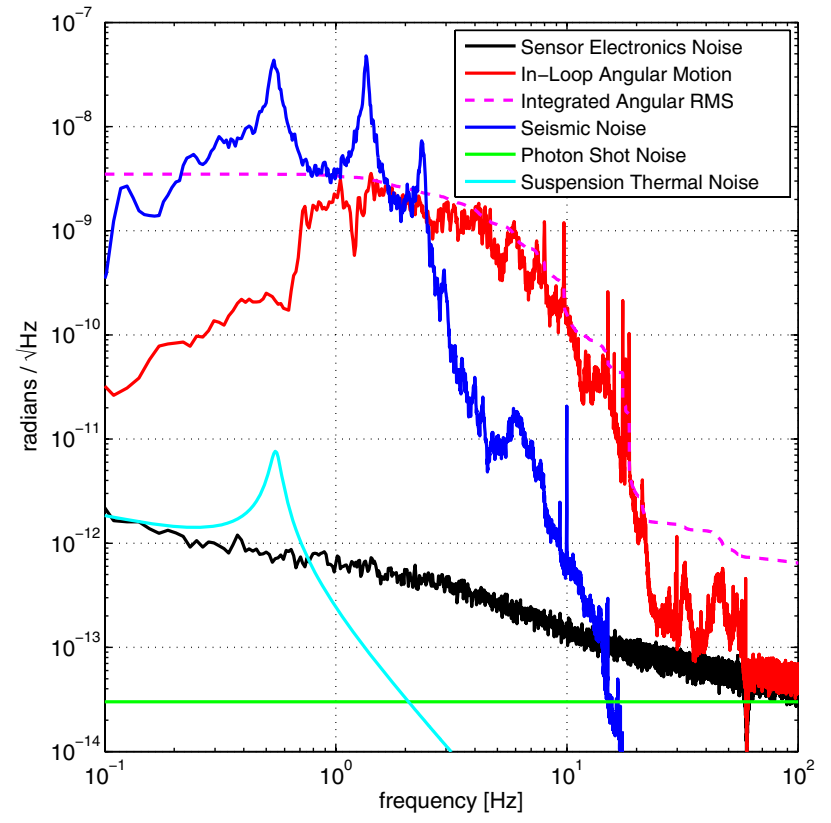

Fig. 2. Noise budget of an alignment sensor (WFS1) for the pitch degree of freedom. Curves for seismic noise, suspension thermal noise, and sensor noise are shown. Note that direct seismic and suspension thermal noises are small in the GW band, and the largest motions are impressed by our control system. Sensing noise dominates above approximately $20 \mathrm{~Hz}$ where the seismic isolation stacks strongly isolate.

coupling, acoustic noise, and radiation pressure torque. Mechanical and electrical design of suspensions and sensors, isolation in vacuum, and periodic balancing of mirror actuators are measures taken to reduce the level of angular motion in the first place. An active control system is used to mediate the motion that remains, which in turn is itself a source of misalignments due to sensing noise. As reflected in the noise budget of one of the alignment sensors in Fig. 2, direct seismic and suspension thermal noises are in fact quite small in the $\mathrm{GW}$ band. Above $20 \mathrm{~Hz}$, where the seismic isolation platforms strongly isolate, sensor noise dominates. As a result, the angular motions of the cavities above these frequencies are dominated by the control system itself. Sensor noise is thus a primary consideration in servo design.

The alignment of the interferometer is accomplished via feedback, and there are several frames of reference to which the mirrors are aligned. Ultimately, the mirrors must be aligned to one another, and this will be presented in detail shortly. Each individual optic also has two servos of its own to provide velocity damping. First, local shadow sensors provide damping around the pitch and yaw eigenfrequencies of the mirrors ( 0.6 and $0.5 \mathrm{~Hz}$, respectively). This damping is relative to the suspension cage, which is already isolated at high frequencies. Second, optical levers mounted to heavy piers on the ground provide a reference to the local ground motion. They are more sensitive than the shadow sensors and serve to suppress the motion which arises from the isolation table stack resonances from 0.2 to $2 \mathrm{~Hz}$. The interaction of these two velocity damping servos with the main alignment servo results in some increased complexity of the main servo design.

The fundamental physical principle behind sensing relative mirror misalignment is the fact that when an optical cavity is misaligned relative to an incident field, a $\mathrm{TEM}_{01}$
Hermite-Gaussian mode is generated with an amplitude proportional to the misalignment [16]. Alignment signals are produced by directing some of this light onto a quadrant photodiode (QPD), where the interference of the $\mathrm{TEM}_{00}$ fundamental mode and $\mathrm{TEM}_{01}$ misalignment mode at the sideband frequency can be compared on each half of the split diode. The QPD together with the resonant $\mathrm{RF}$ circuit and demodulation system is called a wavefront sensor (WFS). The amplitude of the alignment signal is a function of the relative Gouy phase [17] between the $\mathrm{TEM}_{00}$ and $\mathrm{TEM}_{01}$ modes, which is a function of the longitudinal position of the detector along the optical axis. Angular misalignments of different combinations of mirrors can therefore be distinguished by placing detectors at different locations along the optical path. The basic formalism of how alignment signals are generated is presented in [18-20].

A detailed description of the control-scheme design for the Initial LIGO configuration is found in [15], and key aspects relevant for the description of the Enhanced LIGO ASC are provided here. There are eight mirrors whose pitch (rotation about the mirror's horizontal axis) and yaw (rotation about the vertical axis) angles must be sensed and controlled. The sensing is accomplished through the use of eight sensors, which can be organized into three types:

- Wavefront sensors (WFS1, WFS2 [21], WFS3, WFS4), which sense the angular misalignment of the cavities with respect to their input beams

- CCD image of the beam spot on the beam splitter (BS)

- Quadrant photodiodes (QPDX, QPDY), which see the beam transmitted through the arm cavities

Figure 1 shows the basic power-recycled Michelson interferometer layout, highlighting the locations of these angular sensors and the eight mirrors they must control. Two WFS, separated in Gouy phase, are located at the reflected port of the interferometer where common mode signals appear. The third sees a pick-off of light from the recycling cavity, which contains common and differential signals, and the fourth gets a pick-off of the light at the antisymmetric port where differential mode signals are transmitted. The coordinate system defining common and differential motion is found in Table 2. The eight mirrors include the four test masses that make up the Fabry-Perot arm cavities (ITMX, ITMY, ETMX, ETMY), the BS, the recycling mirror (RM), and two input beam directing mirrors that also serve as a mode matching telescope (MMT1 and MMT3).

The CCD image and the QPDs are used in slow feedback loops as part of drift control servos to maintain the beam spot positions at the three corners of the interferometer. Their bandwidths are below a few millihertz and below $0.1 \mathrm{~Hz}$, respectively, and are significantly lower than the bandwidths of the WFS loops, which keep the mirrors aligned to one another from DC up to several Hertz.

Figure 3 shows a simplified block diagram of the WFS servo. The interferometer converts individual mirror motions into optical modes, which, in turn, are converted into error signals by the WFS. The angular error signals are digitized, filtered, and converted into analog control signals for individual mirrors. Two matrices in series rotate the alignment signals from the WFS basis to the optic basis. Control filters are implemented in the intermediate basis. 


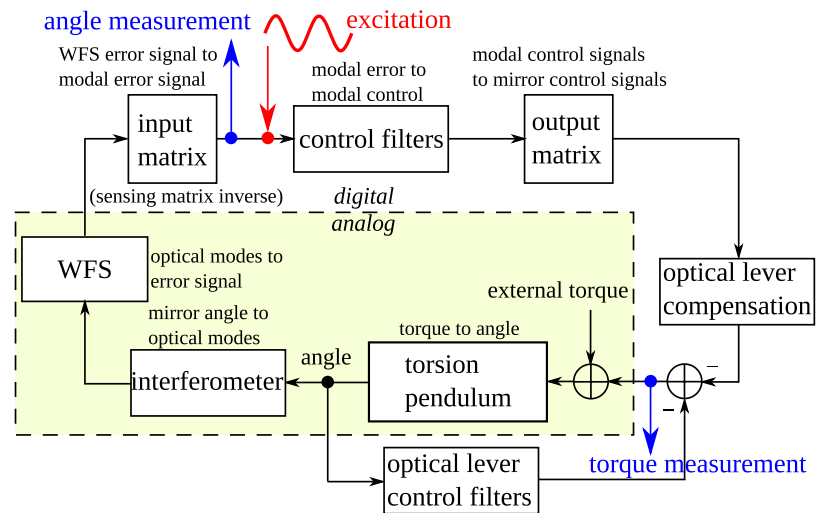

Fig. 3. Block diagram of major components of the angular control servo. The input matrix is the inverse of the sensing matrix presented in Table 3. Components within the dashed box are analog.

In Initial LIGO, the sensing basis was that of common and differential ETM/ITM motion and the RM, and servos were designed in this basis. The input matrix was diagonal, and the output matrix was created to send equal or equal and opposite signals to the ETMs and ITMs, respectively. In this work, we describe a change of basis to improve the stability of the interferometer in the presence of radiation pressure torque.

\section{ASC DESIGN IN THE PRESENCE OF RADIATION-PRESSURE INSTABILITY}

The effectiveness of the Initial LIGO ASC design is limited in the regime of high circulating power where radiation pressure modifies the simple pendulum plant in a way that is powerdependent. As is detailed in this section, torque due to radiation pressure couples the angular motions of the arm cavity mirrors such that the simple single resonance of a given mirror's torque-to-angle transfer function splits into two, with frequency shifts dependent on power. Controlling this new plant could be accomplished with the Initial LIGO system by increasing the gains of the WFS loops, but it would be at the expense of introducing too much control noise in the GW measurement band. An alternative solution is thus required to achieve both adequate angular control and minimal noise impression. In this section, we first review the formalism of radiation-pressure torque in cavities. Then we present a direct measurement of the opto-mechanical modes of the Enhanced LIGO arm cavities for several powers. Finally, we describe the modified control scheme and present its implementation.

\section{A. Torque Induced by Radiation Pressure}

In the limit of no circulating power in a suspended FabryPerot cavity, each of the individual mirrors has independent equations of motion. With power circulating in the cavity, however, radiation pressure effects couple the equations of motion of the two mirrors. As a beam impinging a mirror off-center creates torque, an opto-mechanical angular spring is created due to the geometric relationship of beam displacements and mirror angles [17]. This fact has two important consequences: on one hand, as the torque induced by radiation pressure is proportional to the power stored inside the cavity, the opto-mechanical angular transfer functions of the cavity mirrors change as a function of the stored power. On the other hand, for large powers, radiation pressure can even overcome the restoring torque of the mirror suspension, creating an unstable system.

To understand how the cavity dynamics are affected by radiation pressure, it is useful to diagonalize the coupled equations of the mirror motion into two normal cavity modes. We refer to [9] for a complete derivation of the torsional stiffness matrix, which couples the static misalignment of the two cavity mirrors; here we use only the final expressions for the two eigenvalues $k_{S, H}$ and eigenvectors $v_{S, H}$ of that matrix

$$
\begin{gathered}
k_{S, H}=k_{0} \frac{\left(g_{1}+g_{2}\right) \pm \sqrt{\left(g_{1}-g_{2}\right)^{2}+4}}{2}, \\
v_{S}=\left[1, \frac{k_{0}}{k_{S}-k_{0} g_{1}}\right]
\end{gathered}
$$

$$
v_{H}=\left[\frac{k_{0}}{k_{0} g_{2}-k_{H}}, 1\right],
$$

where $k_{0}=\left(2 P L / c\left(g_{1} g_{2}-1\right)\right) \quad(L=3995 \mathrm{~m}$ is the cavity length, $c$ the speed of light, and $g_{1}$ and $g_{2}$ the geometric $g$ factors of the cavity).

The resonant frequency of each of the opto-mechanical modes can then be written as

$$
f_{S, H}=\frac{1}{2 \pi} \sqrt{\frac{k_{p}+k_{S, H}}{I}}
$$

where $I$ is the mirror moment of inertia $\left(I=0.0507 \mathrm{~kg} \mathrm{~m}^{2}\right)$, and $k_{p}$ is the restoring torque of the mirror suspension $\left(k_{p}=0.72 \mathrm{Nm} / \mathrm{rad}\right.$ pitch and $0.5 \mathrm{Nm} / \mathrm{rad}$ yaw). For the Initial and Enhanced LIGO interferometers, the $g$ factors of the cavities are

$$
\begin{gathered}
g_{1}=g_{\mathrm{ITM}}=1-L / R_{\mathrm{ITM}}=0.726, \\
g_{2}=g_{\mathrm{ETM}}=1-L / R_{\mathrm{ETM}}=0.460,
\end{gathered}
$$

so $k_{S}$ is negative and $k_{H}$ is positive.

Known as the Sidles-Sigg effect, the radiation pressure torque either softens or stiffens the mechanical springs. We therefore refer to the two modes as "soft (S)" or "hard (H)." As power increases, the frequency of the hard mode increases, but the frequency of the soft mode decreases until $k_{S}+k_{p}<0$ when there is no longer a real resonant frequency, corresponding to an unstable system.

The values of the opto-mechanical frequencies of the soft and hard modes for Initial and Enhanced LIGO powers are outlined in Table 1 . The increase of stored power from $9 \mathrm{~kW}$ in Initial LIGO to $40 \mathrm{~kW}$ in Enhanced LIGO makes

Table 1. Resonant Frequencies (Pitch) in Hertz for Soft and Hard Opto-Mechanical Modes ${ }^{a}$

\begin{tabular}{lcccc}
\hline & $P_{\text {circ }}[\mathrm{kW}]$ & $f_{p}[\mathrm{~Hz}]$ & $f_{S}[\mathrm{~Hz}]$ & $f_{H}[\mathrm{~Hz}]$ \\
\hline Initial LIGO & 9 & 0.60 & 0.19 & 0.66 \\
Enhanced LIGO & 40 & 0.60 & -1.04 & 0.83 \\
\hline
\end{tabular}

${ }^{a}$ Frequencies shown are for the typical Initial LIGO circulating power $(9 \mathrm{~kW})$ and the highest of Enhanced LIGO powers $(40 \mathrm{~kW})$. The soft mode in Enhanced LIGO is unstable. 
radiation-pressure torque cross into the realm of significance. The torque due to radiation pressure surpasses the suspension-restoring torque such that the soft opto-mechanical mode, which had just approached instability in Initial LIGO, actually becomes unstable in Enhanced LIGO.

\section{B. Measurement of Opto-Mechanical Modes}

We directly measured the soft and hard modes for several different powers, as presented in Fig. 4, where solid curves are fits of the data to the Sidles-Sigg model. By injecting an excitation into the control leg of the servo loop and taking the transfer function from the torque input to the resulting angle as measured by the WFS (in the radiation-pressure eigenbasis), we reproduce the transfer function of the optomechanical plant, independent of the control system. The measurement points are highlighted in Fig. 3. Note that for the measurement to be independent of the control system, the control system must be perfectly diagonalized, so that it acts as a collection of single-input single-output loops. Although we periodically tuned the input matrix to keep the system diagonal, some cross-coupling is expected.

The circulating powers listed in the figure legend are calculated as follows:

$$
P_{\text {circ }}=P_{\text {in }} \varepsilon g_{\mathrm{PRC}} T_{\mathrm{BS}} g_{\phi},
$$

where $\varepsilon=0.7$ is the optical efficiency of the optics between the laser and interferometer, $g_{\mathrm{PRC}}=35$ is the power gain of the power-recycling cavity, $T_{\mathrm{BS}}=0.5$ is the transmission of the BS, $g_{\phi}=137$ is power gain of the Fabry-Perot arms, and $P_{\text {in }}$ is the measured input power. Our error in the estimation of circulating power is $\pm 20 \%$.

Figure $\underline{4(a)}$ shows the measurements of the soft optomechanical transfer function for four different circulating powers. As power increases from 1.7 to $10 \mathrm{~kW}$, we observe a decrease in the soft-mode resonant frequency from 0.6 to $0.4 \mathrm{~Hz}$. As the circulating power is increased to $17 \mathrm{~kW}$ and beyond, the resonance disappears as expected: the plant has become statically unstable. Likewise, as shown in Fig. 4(b), we measured the anticipated increase in resonant frequency as a function of power for the hard mode. The resonant frequency increases from 0.66 to $0.95 \mathrm{~Hz}$ as the power increases from 1.7 to $17 \mathrm{~kW}$. We confirm that the plant for which controls must be designed is no longer that of a pendulum with a resonance at $0.6 \mathrm{~Hz}$ (pitch) or $0.5 \mathrm{~Hz}$ (yaw), but that of the soft and hard opto-mechanical system.

\section{High Laser Power Angular Controls}

We describe an alignment scheme for controlling the mirrors with radiation-pressure-dominated angular dynamics, which make use of the elegant implication of the purely geometric description of a cavity mode [see Eq. (4)]; the opto-mechanical degrees of freedom remain uncoupled, independent of the circulating power. This is the core of the ASC work for Enhanced LIGO: to create an input matrix to rotate the WFS control to the basis of common and differential opto-mechanical eigenmodes, implement filters in this newly formed basis, and then increase the gains of only those loops that require it [13].
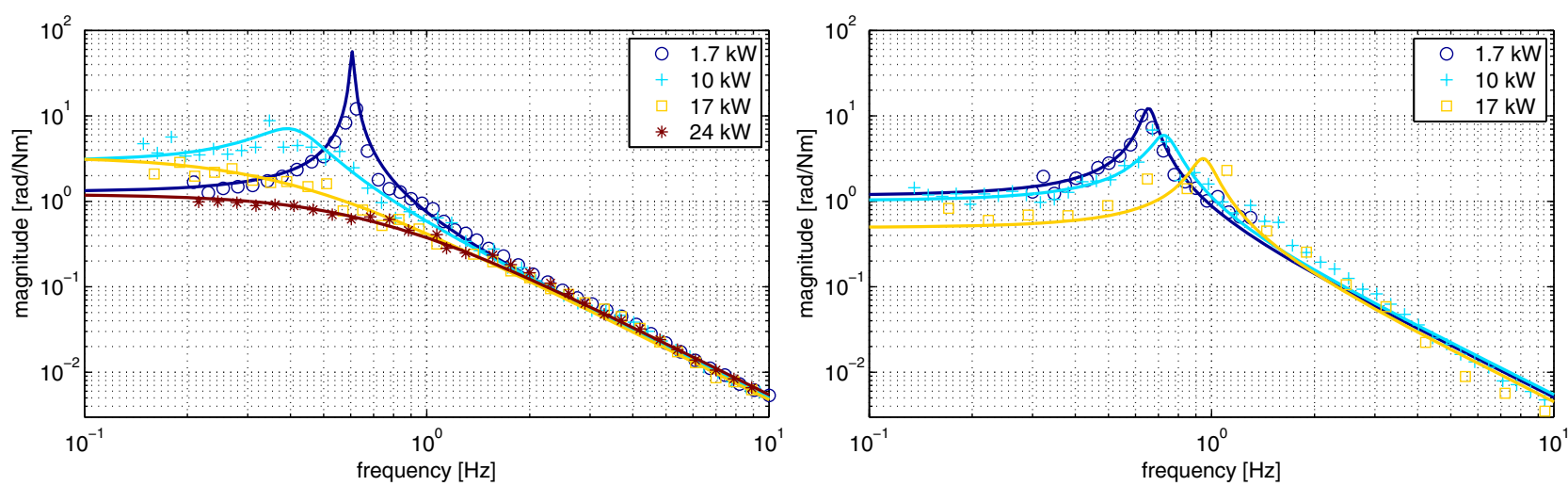

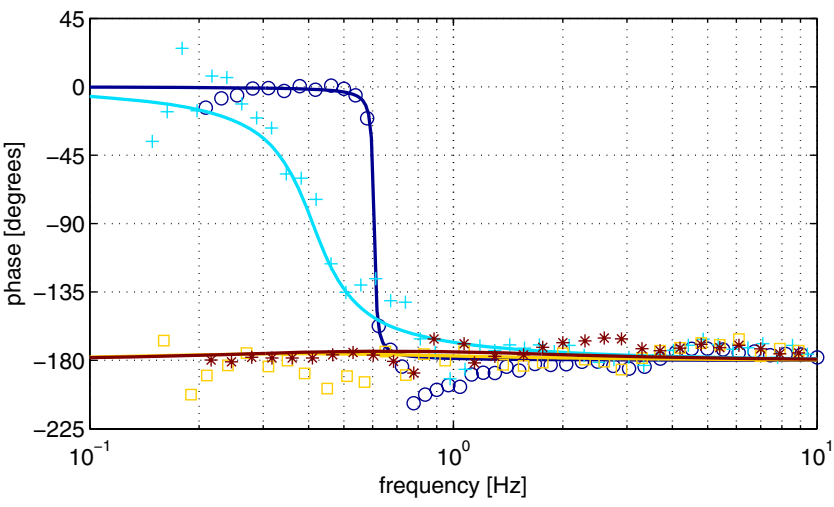

(a)

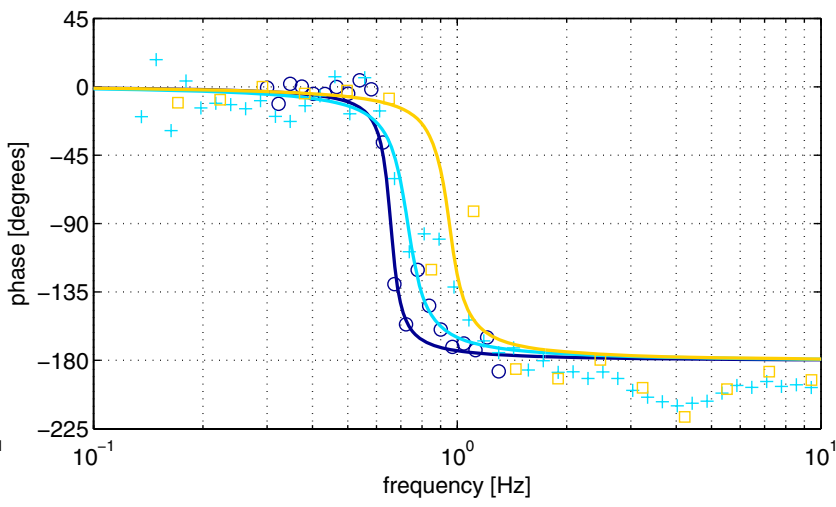

(b)

Fig. 4. Measured opto-mechanical transfer functions at different powers for the (a) soft and (b) hard degrees of freedom. The resonant frequency increases with power for the hard mode and decreases with power for the soft mode, which eventually becomes unstable. $P_{\text {circ }}=9 \mathrm{~kW}$ was a typical operating power for Initial LIGO, and $P_{\text {circ }}=40 \mathrm{~kW}$ is the highest of powers reached for Enhanced LIGO. Solid curves indicate fits to the measured data points. 
The five controlled opto-mechanical degrees of freedom are as follows: differential soft (dSoft), common soft (cSoft), differential hard (dHard), common hard (cHard), and recycling mirror (RM).

Each of the two Fabry-Perot cavity arms has a soft and a hard mode. The use of common/differential degrees of freedom is motivated by the block-diagonal structure of the sensing matrix in this basis. The common mode represents motion where the optics of one FP cavity rotate in the same direction as the other cavity and differential represents rotations in opposite directions. The recycling mirror is sensed separately.

The servos that actively control the soft degrees of freedom must be designed to provide overall stability. This is, in fact, not difficult because the decreasing resonance naturally moves deeper into the control bandwidth toward frequencies where gain is higher. At powers when the resonance disappears, only DC control is necessary. Counterintuitively, it is the hard, stable mode that poses the greater control challenge. As the resonance of the hard mode increases with power, it has the potential of making the overall control loop unstable. The control loop therefore requires a higher bandwidth than that for the soft mode to be stable and always provide damping of the hard mode resonant frequency.

Moreover, the maximum laser power stored inside the LIGO cavities is reached by progressively increasing the input power. Although the soft and hard modes remain uncoupled, the servo loops still need to provide stability over their respective wide ranges of opto-mechanical transfer functions.

The structure of the angular control loop is to use input and output matrices before and after the control filters, respectively. The input matrix realizes the change of basis from the WFS sensors to the opto-mechanical eigenmodes, and the output matrix changes the basis once again, from the eigenmodes to individual mirrors. The output matrix is shown in Table 2. It is the analytical basis transformation matrix that diagonalizes the coupled equations of motion [see Eq. (4)] and is repeated with appropriate sign changes to form differential and common soft and hard modes of the two arms. The matrix is arbitrarily normalized, so the largest element is 1 , and $r$ is 0.91 for Livingston and 0.87 for Hanford (a result of different mirror radii of curvature at each site).

The input matrix is determined experimentally by measuring its inverse, a sensing matrix that details the specific combinations of WFS which sense the hard and soft modes. A calibrated sensing matrix for the radiation-pressure eigenbasis as taken during a $17 \mathrm{~kW}$ lock is shown in Table $\underline{3}$. Rows represent hard/soft eigenmode excitation, and columns are the WFS signals. Before inverting the sensing matrix to create

Table 2. WFS Output Matrix for Pitch (Yaw) ${ }^{a}$

\begin{tabular}{cccccc}
\hline dSoft & dHard & cSoft & cHard & RM & \\
\hline$+(+) 1$ & $+(+) r$ & $+(+) 1$ & $+(+) r$ & 0 & ETMX \\
$-(+) 1$ & $-(+) r$ & $+(-) 1$ & $+(-) r$ & 0 & ETMY \\
$+(-) r$ & $-(+) 1$ & $+(-) r$ & $-(+) 1$ & 0 & ITMX \\
$-(-) r$ & $+(+) 1$ & $+(+) r$ & $-(-) 1$ & 0 & ITMY \\
& 0 & 0 & 0 & 1 & RM \\
\hline
\end{tabular}

${ }^{a}$ The geometry of the arm cavities dictates the necessary relative magnitudes of actuation on each mirror to create the soft and hard modes. A positive yaw moves each mirror clockwise when viewed from above; a positive pitch tips the tops of the mirrors in a cavity toward each other when viewed from the side. For Livingston $r=$ 0.91 and for Hanford $r=0.87$.
Table 3. Sensing Matrix in Units of [V/rad] (Pitch $)^{a}$

\begin{tabular}{|c|c|c|c|c|c|}
\hline WFS1Q & WFS2Q & WFS2I & WFS3I & WFS4I & \\
\hline 2.0 & 0.03 & 0.06 & -0.008 & 0.01 & dSoft \\
\hline 0.31 & -0.03 & -0.04 & 0.002 & -0.01 & dHard \\
\hline 0.02 & -0.01 & 0.18 & -0.02 & -0.10 & cSoft \\
\hline 0.17 & -0.01 & -0.21 & 0.007 & -0.12 & cHard \\
\hline 0.09 & -0.01 & -0.21 & 0.04 & -0.21 & $\mathrm{RM}$ \\
\hline
\end{tabular}

${ }^{a}$ All elements are measured at $9.7 \mathrm{~Hz}$ in the closed-loop system, but with the feedback at $9.7 \mathrm{~Hz}$ notched out. Numbers in gray are the measurement results that have coherence less than 0.9 . Boxes highlight the elements actually used for computing the control servo's input matrix (inverse of sensing matrix); all other elements are set to zero.

the input matrix, the smallest of the elements (which are equivalent to the elements for which optical gain is expected to be weak) are set to zero. The elements that remain are highlighted by boxes. Note that the sensing matrix is in fact composed of two submatrices: one for the differential degrees of freedom, and one for the common degrees of freedom. Also, due to geometric reasons, WFS1Q has a particularly strong signal compared to the other WFS, which allows us to provide more control to the dSoft mode than to the other modes.

\section{RESULTS}

In this section we present measurements of the performance of the ASC system with up to $27 \mathrm{~kW}$ circulating power and demonstrate that the ASC design meets the LIGO requirements.

\section{A. Open-Loop Gain}

The open-loop transfer function of each of the WFS loops is the product of the radiation-pressure-modified pendulum and the control filters. Figure 5 shows the open-loop transfer functions of each of the WFS loops as measured during a
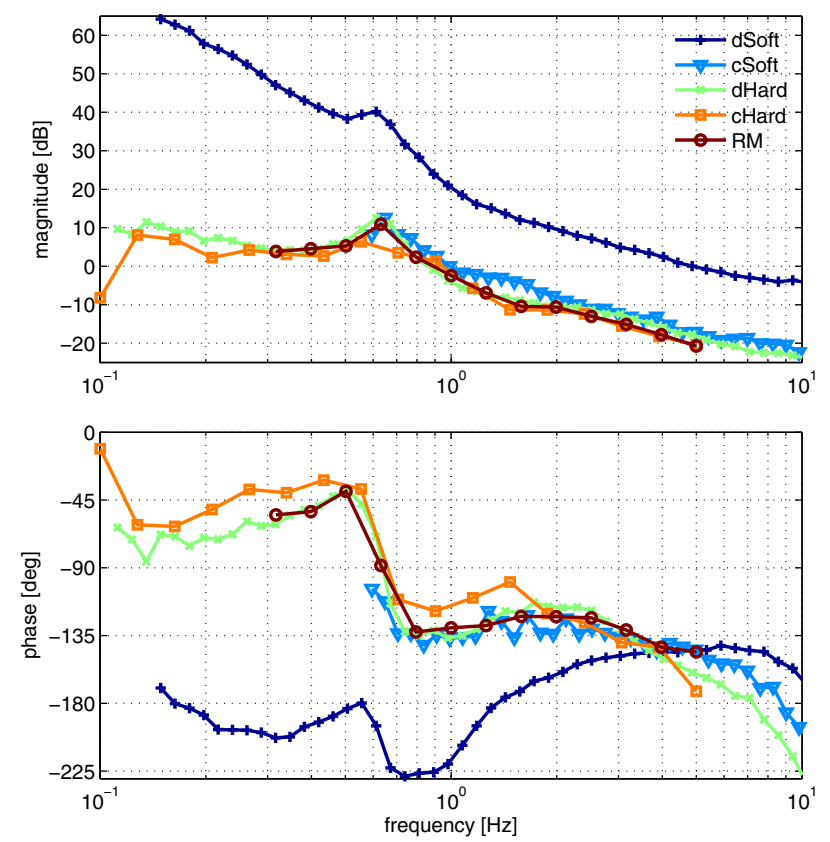

Fig. 5. Open-loop gains (pitch) of the five WFS loops as measured with $10 \mathrm{~kW}$ circulating power. All have a phase margin of $40^{\circ}$ to $50^{\circ}$. All UGFs are around $1 \mathrm{~Hz}$ with the exception of the dSoft degrees of freedom whose UGF is $5 \mathrm{~Hz}$. 
$10.3 \mathrm{~kW}$ lock with the loops closed. As anticipated from the large dSoft signal seen by WFS1 in the sensing matrix measurement (Table 3), that is the mode for which we can and do provide the strongest suppression. In order to achieve this much suppression, it is necessary to make the feedback loop conditionally stable. As shown here, the dSoft unity gain frequency (UGF) is at $5 \mathrm{~Hz}$. All of the other degrees of freedom have UGFs of $\sim 1 \mathrm{~Hz}$ and are designed to be unconditionally stable.

The dSoft loop could have a higher gain compared to the other loops because it caused no harm in strain sensitivity above $60 \mathrm{~Hz}$, as is presented later in Section 4.C. The UGFs of all other loops were selected as a necessary minimum.

\section{B. Residual Beam Spot and Mirror Motion}

Figure 6 shows spectra of the residual angular motion in each of the eigenbasis degrees of freedom during a $17 \mathrm{~kW}$ lock. The typical residual $\mathrm{rms}$ angular motion is $10^{-7} \mathrm{rad} / \sqrt{\mathrm{Hz}}$.

Above $20-25 \mathrm{~Hz}$, the WFS signals do not represent true angular motion but instead are limited by a combination of optical shot noise, photodetector electronics noise, and acoustic noise. Unless sufficiently filtered, the control signal derived from frequencies in this band will increase the mirror motion. The resulting need for low-pass filters limits the achievable bandwidth of the loops. Because reducing the design UGF allows us to reduce the corner frequency of the (steep) low-pass filters, the reduction of noise in the GW band is inversely proportional to the UGF raised to the third or even fourth power.

The residual beam spot motion on the test masses is shown in Fig. 7. The rms beam spot motion on the ETMs is $1 \mathrm{~mm}$, and on the ITMs it is $0.8 \mathrm{~mm}$. These measurements are acquired from the pitch and yaw signals of the QPDs in transmission of
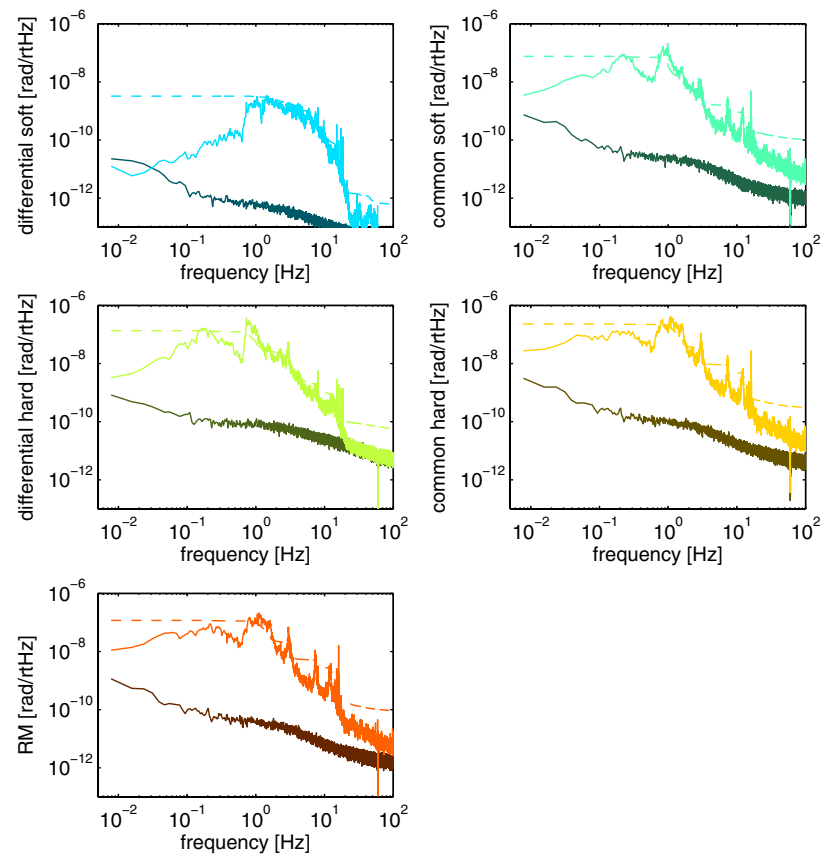

Fig. 6. Residual motion of the opto-mechanical degrees of freedom during a $17 \mathrm{~kW}$ lock. Dashed lines are the rms integrated from the right; note that the rms is dominated by the approximately $1 \mathrm{~Hz}$ pendular motion. Sensor noise represented in the opto-mechanical eigenbasis is also shown.

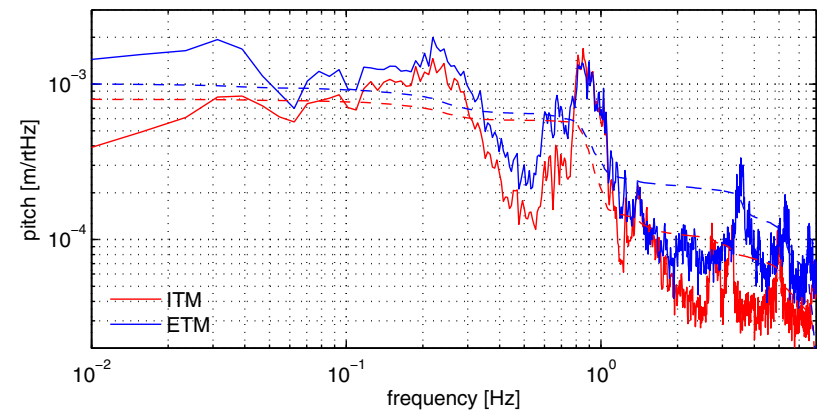

Fig. 7. Beam spot motion (pitch) on the ITM and ETM mirrors during a $27 \mathrm{~kW}$ lock at night. Dashed lines are the integrated spectral density. For pitch and yaw, the rms beam spot motion is $1 \mathrm{~mm}$ on the ETMs and $0.8 \mathrm{~mm}$ on the ITMs.

the ETMs and the pitch and yaw DC signal from WFS2 for the ITMs. The magnitudes of the beam spot motion and the residual mirror motion are consistent. For example, for $10^{-7} \mathrm{rad}$ of soft or hard mode motion in one arm, we expect the maximum cavity tilt and displacement to be $0.1 \mathrm{rad}$ and $1 \mathrm{~mm}$, respectively.

\section{Angle-to-Length Coupling (Noise Performance)}

One of the most important figures of merit for the control system is how much noise it contributes to the GW strain signal. As described in Section 2, the dominant way in which angular motion creates a change in cavity length is the convolution of beam spot motion with angular mirror motion. Ideally, we want the length displacement due to this coupling to be an order of magnitude below the desired displacement sensitivity.

The effective transfer function magnitude of the angleto-length effects can be estimated with a broadband noise injection that amplifies the mirror motion. This nonlinear technique is necessary because the linear coupling of torque to cavity length is minimized by periodically balancing the mirror actuators. Due to the near-elimination of the linear coupling, the remaining dominant angle-to-length process [refer to Eq. (1)] has a coupling coefficient of mean 0, and the traditional coherent transfer function measurement would therefore also yield 0 . To arrive at an estimate of the magnitude of the remaining time-dependent angle-to-length coupling, the broadband excitation must be averaged over some time. We injected a 40 to $110 \mathrm{~Hz}$ broadband excitation into the error point after the input matrix and computed a transfer function between the hard/soft eigenmode error point and the GW signal. The transfer function may be multiplied by an ASC signal at any time to estimate a noise budget.

The WFS noise budget in the eigenmode basis for pitch at a time when the interferometer was locked with $24 \mathrm{~kW}$ power is shown in Fig. 8(a). Each degree of freedom's contribution of control noise to displacement sensitivity is the same within about a factor of 2, except for the RM, which is not included in these plots. We were not able to measure the transfer function for RM motion to displacement sensitivity because so large of an excitation was required to see an effect that the interferometer would lose lock. The soft modes contribute more length noise than the hard modes.

The ASC is, in fact, the limiting noise source for frequencies up to $55 \mathrm{~Hz}$, and it becomes less and less of a primary noise source as frequency increases. By $100 \mathrm{~Hz}$, the ASC noise floor 


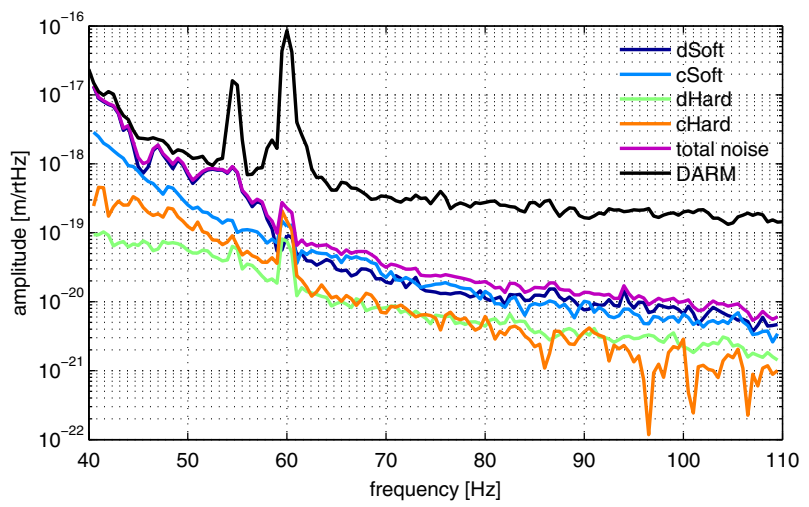

(a)

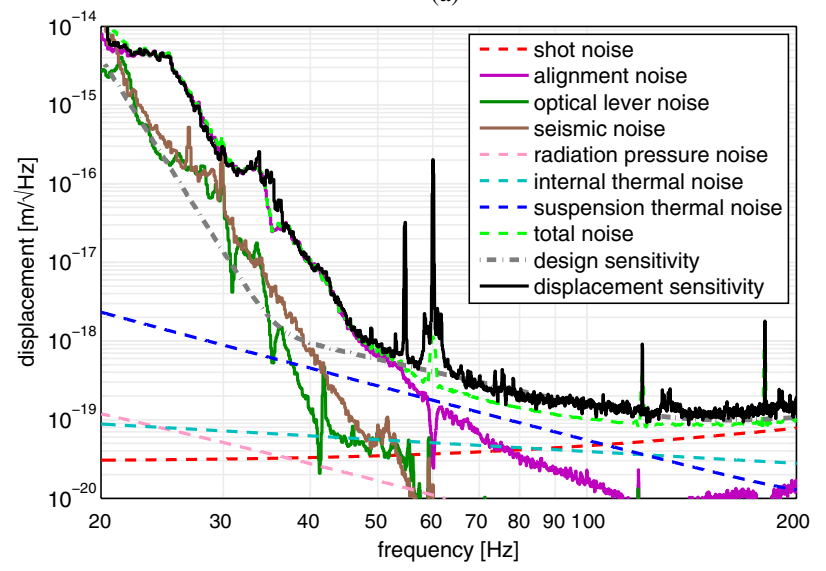

(b)

Fig. 8. Displacement sensitivity noise budget during a lock with $24 \mathrm{~kW}$ circulating power. (a) Break down of the noise budget of the alignment feedback (pitch) degrees of freedom to displacement sensitivity. The two soft modes contribute more than the hard modes. The RM alignment feedback is not shown because its contribution is insignificant. (b) Noise budget of interferometer displacement sensitivity, showing several key noise sources. Angular control limits the sensitivity up to $55 \mathrm{~Hz}$. Pitch and yaw contributions are added in quadrature because they are decoupled.

is a factor of 10 below displacement sensitivity. The specific structure of the noise contributions, including the apparent notches, is a direct result of the shape of the control filters. Imperfections in the estimate of displacement noise below $50 \mathrm{~Hz}$ arise because the transfer function is not perfectly stable in time.

Figure $8(\mathrm{~b})$ shows a broader view of the role of angular control noise with respect to other primary noise sources. The alignment noise shown is the quadrature sum of the pitch and yaw contributions. Measured seismic and optical lever noises are also shown, in addition to models of thermal, shot, and radiation-pressure noises. In this example, ASC noise hinders the interferometer sensitivity up to $55 \mathrm{~Hz}$ by about an order of magnitude. At a later time, steeper-and lowerfrequency low-pass control filters were made (at the expense of reduced stability) to reduce the alignment noise to a level similar to that of seismic noise.

\section{ADVANCED LIGO ALIGNMENT CONSIDERATIONS}

The LIGO detectors are currently being upgraded to a configuration known as Advanced LIGO to achieve up to a factor of 10 improvement in broadband sensitivity [22]. The noise

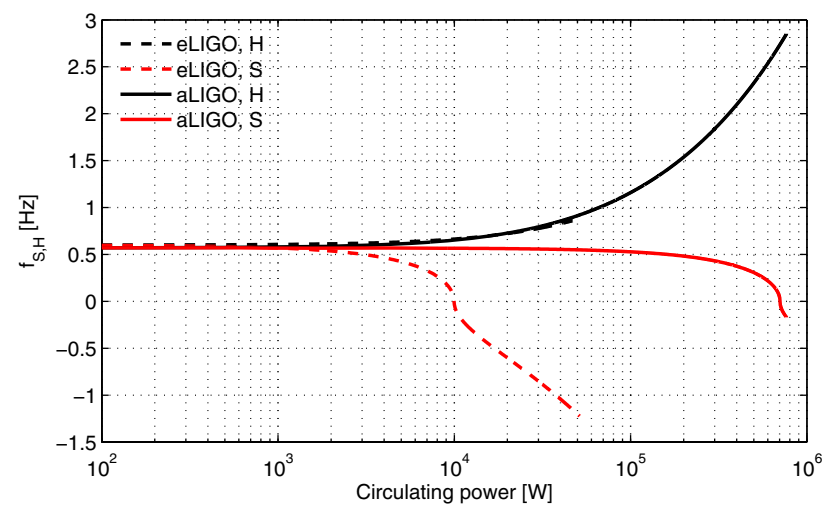

Fig. 9. Resonant frequency of the opto-mechanical modes for pitch as a function of circulating power, comparing Advanced and Enhanced LIGO. Only at the highest of Advanced LIGO powers (about $800 \mathrm{~kW}$ circulating power, or $125 \mathrm{~W}$ input power) will the soft mode become unstable. Models are plotted up to the highest of their respective design powers.

performance of the angular control scheme in the Advanced LIGO detectors must meet the most stringent requirements to date, as imposed by the improved sensitivity and the goal that the displacement noise produced by the ASC is no greater than $10 \%$ of the design sensitivity. Given the ASC was a limiting noise source below $55 \mathrm{~Hz}$ in Enhanced LIGO, some additional steps must be taken to achieve the Advanced LIGO goal. For instance, in order to mitigate the largely acousticdominated WFS noise above $10 \mathrm{~Hz}$, the WFS will be placed in vacuum for Advanced LIGO. In addition, the angle-to-length coupling at low frequencies will be reduced through the use of a seismic feed-forward scheme.

The Sidles-Sigg effect will not be as important in Advanced LIGO despite the laser power stored in the arm cavities being as high as $800 \mathrm{~kW}, 20$ times higher than in Enhanced LIGO. A number of design changes have made the impact of radiation pressure less dramatic: four times heavier mirrors (40 kg instead of $10 \mathrm{~kg}$ ); arm cavity $g$ factor chosen to suppress the soft mode [9]; and a larger restoring angular torque due to new multi-stage pendulum suspensions.

Figure 9 shows a plot of soft- and hard-mode frequency as a function of stored power in the arms for the Enhanced and Advanced LIGO configurations. It can be seen that, although the hard mode is hardly affected by the Advanced LIGO changes, the new $g$ factor greatly pushes out the power at which the soft mode becomes unstable. Nevertheless, the control strategy developed for Enhanced LIGO gives us confidence that we can control the hard and soft modes. The Advanced LIGO ASC design detailing the effects of the above changes to the design presented here is found in [23].

\section{CONCLUSION}

The Enhanced LIGO interferometer is a complex optomechanical system whose angular mechanics are dominated by radiation-pressure effects. We show that radiation pressure shapes the angular dynamics of the suspended mirrors and plays an important role in the design of an angular control system. We implemented and characterized a novel control scheme to deal with the instabilities that radiation pressure causes to the angular degrees of freedom of the interferometer, without compromising the strain sensitivity of the detector. The alignment control scheme that we describe allowed 
the LIGO detectors to operate at their best sensitivity ever, as achieved during scientific run S6. The solution that we demonstrate here is extensible to the next generation of LIGO detectors, Advanced LIGO, and is more broadly applicable in systems in which radiation pressure torques are dominant over mechanical restoring forces.

\section{ACKNOWLEDGMENTS}

We would like to thank D. H. Reitze, D. Sigg, Y. Aso, G. Mueller, and H. Grote for fruitful discussions. LIGO was constructed by the California Institute of Technology and Massachusetts Institute of Technology with funding from the United States National Science Foundation under grant PHY-0757058. K. L. Dooley recieved support from the National Science Foundation under grant PHY-0855313. This paper has LIGO document number P1100089.

\section{REFERENCES AND NOTES}

1. R. X. Adhikari, "Gravitational radiation detection with laser interferometry," 2013, http://arxiv.org/abs/1305.5188.

2. B. P. Abbott, et al. "LIGO: the Laser Interferometer GravitationalWave Observatory," Rep. Prog. Phys. 72, 076901 (2009).

3. R. Adhikari, P. Fritschel, and S. Waldman, "Enhanced LIGO," Tech. Rep. T060156 (LIGO Laboratory, 2006).

4. M. Frede, B. Schulz, R. Wilhelm, P. Kwee, F. Seifert, B. Willke, and D. Kracht, "Fundamental mode, single-frequency laser amplifier for gravitational wave detectors," Opt. Express 15, 459-465 (2007).

5. T. Fricke, N. Smith-Lefebvre, R. Abbott, R. Adhikari, K. Dooley, M. Evans, P. Fritschel, V. Frolov, K. Kawabe, J. Kissel, B. Slagmolen, and S. Waldman, "DC readout experiment in Enhanced LIGO," Class. Quantum Grav. 29, 065005 (2012).

6. K. L. Dooley, M. A. Arain, D. Feldbaum, V. V. Frolov, M. Heintze, D. Hoak, E. A. Khazanov, A. Lucianetti, R. M. Martin, G. Mueller, O. Palashov, V. Quetschke, D. H. Reitze, R. L. Savage, D. B. Tanner, L. F. Williams, and W. Wu, "Thermal effects in the input optics of the Enhanced Laser Interferometer Gravitational-Wave Observatory interferometers," Rev. Sci. Instrum. 83, 033109 (2012).
7. S. Solimeno, F. Barone, C. de Lisio, L. Di Fiore, L. Milano, and G. Russo, "Fabry-Pérot resonators with oscillating mirrors," Phys. Rev. A 43, 6227-6240 (1991).

8. J. A. Sidles and D. Sigg, "Optical torques in suspended FabryPerot interferometers," Tech. Rep. P030055-B (LIGO Laboratory, 2003).

9. J. Sidles and D. Sigg, "Optical torques in suspended Fabry-Perot interferometers," Phys. Lett. A 354, 167-172 (2006).

10. Y. Fan, L. Merrill, C. Zhao, L. Ju, D. Blair, B. Slagmolen, D. Hosken, A. Brooks, P. Veitch, D. Mudge, and J. Munch, "Observation of optical torsional stiffness in a high optical power cavity," Appl. Phys. Lett. 94, 081105 (2009).

11. J. Driggers, "Optomechanical alignment instability in LIGO mode cleaners," Tech. Rep. T060240-00-R (LIGO Laboratory, 2006).

12. E. Hirose, K. Kawabe, D. Sigg, R. Adhikari, and P. R. Saulson, "Angular instability due to radiation pressure in the LIGO gravitational-wave detector," Appl. Opt. 49, 3474-3484 (2010).

13. L. Barsotti and M. Evans, "Modeling of alignment sensing and control for Enhanced LIGO," Tech. Rep. T080186 (LIGO Laboratory, 2009).

14. P. Fritschel and D. Shoemaker, "Alignment sensing/control design requirements document,” Tech. Rep. T952007 (LIGO Laboratory, 1997).

15. P. Fritschel, N. Mavalvala, D. Shoemaker, D. Sigg, M. Zucker, and G. González, "Alignment of an interferometric gravitational wave detector," Appl. Opt. 37, 6734-6747 (1998).

16. D. Z. Anderson, "Alignment of resonant optical cavities," Appl. Opt. 23, 2944-2949 (1984).

17. A. E. Siegman, Lasers, 1st ed. (University Science Books, 1986).

18. Y. Hefetz, N. Mavalvala, and D. Sigg, "Principles of calculating alignment signals in complex resonant optical interferometers," J. Opt. Soc. Am. B 14, 1597-1605 (1997).

19. E. Morrison, B. J. Meers, D. I. Robertson, and H. Ward, "Automatic alignment of optical interferometers," Appl. Opt. 33 5041-5049 (1994).

20. N. M. Sampas and D. Z. Anderson, "Stabilization of laser beam alignment to an optical resonator by heterodyne detection of off-axis modes," Appl. Opt. 29, 394-403 (1990).

21. Two signals are derived from WFS2.

22. G. M. Harry, and the LIGO Scientific Collaboration, "Advanced LIGO: the next generation of gravitational wave detectors," Class. Quantum Grav. 27, 084006 (2010).

23. L. Barsotti, M. Evans, and P. Fritschel, "Alignment sensing and control in advanced LIGO," Class. Quantum Grav. 27, 084026 (2010). 